\title{
Dominant Pole Based Approximation for Discrete Time System
}

\author{
Richa*, Awadhesh Kumar ${ }^{\#}$ \\ Department of Electrical Engineering \\ Madan Mohan Malaviya University of Technology, Gorakhpur, India \\ E-mails: "rch1163@gmail.com, ${ }^{\#}$ akee@ mmmut.ac.in \\ ${ }^{*}$ Corresponding author
}

(Received March 11, 2018; Accepted November 3, 2018)

\begin{abstract}
This paper presents an effective procedure for model order reduction of discrete time control system. The exact model derived from complex dynamic systems proves to be very complicated for analysis, control and design. This necessity brings about using a tool known as model order reduction technique or model simplification. A novel mixed method has been implemented in this paper for reducing the order of the large scale dynamic discrete system. Dominant pole based pole clustering method has been used to derive the coefficients of denominator polynomial while Padé approximation has been applied to obtain the coefficients of numerator polynomial of the reduced order model. The proposed method is quite simple and able to generate a stable reduced order model from high order stable discrete systems. The dominancy of poles has been decided by values of the ratio of residue to its pole. The pole is considered dominant which have larger ratio value. An illustrative example has been considered to show the various reduction steps. The result obtained confirms the effectiveness of the approach.
\end{abstract}

Keywords-Dominant Pole, Padé approximant, Pole clustering, Reduced order model, Residue based pole clustering.

\section{Introduction}

Behind any mathematical modelling, there is some real world problem and the analysis is often too tedious and costlier due to its complexity. Through reduction procedure, we get a compact or compressed description of behaviour by reducing the complexity of the model by using only the dominant part of the behaviour. Our core objective is to preserve the system properties and ensure a good approximation of the original system and efficient algorithm should be used. The reason why we want to use this approximation is the major question and then we will carry out an example. The main two reasons are they are mathematical in nature and trying to do analysis with reduced model is much easier than a transfer function has a higher order system. The second reason is the presence of FOPDTs tuning, a system with the higher order can be problematic in tuning procedure with different controllers. Large scale order reduction procedures are done to lessen the volume of data for simpler calculation and low response time. We must confirm towards conserving reduced order model stability by the original ones with the efficient procedure and must matches some quantities and responses that are important for the application (Shamash, 1974). In this paper, scheme for the higher order reduction of discrete time systems using Padé approximation and modified pole clustering technique has been proposed (Bistritz and Shaked, 1984; Vishwakarma and Prasad, 2008). By applying linear transformation the original discrete time system is changed to continuous time system and the reduced order model is derivative of the original system by using the above stated techniques which imitates the behaviour of the original one (Ramesh, et al., 2017). Finally, result of the inverse transformation of the derivative of the continuous original system produces reduced order model in discrete time system. 
International Journal of Mathematical, Engineering and Management Sciences

Vol. 4, No. 1, 56-65, 2019

https://dx.doi.org/10.33889/IJMEMS.2019.4.1-005

In arriving at the stable reduced order models, various methods based on the retention of dominant poles have been proposed; roots lying near the unit circle in the Z-plane have more effects on the system response than the others. And those roots are named as dominant roots whereas insignificant roots are lying close the origin. Roots closer to the origin are less important from the maximum overshoot and damping point of view than the dominant roots and these cannot be completely excluded since the excess no. of poles over zeros has a delay effect in initial region of the time response. The dominancy of poles is not decided by the conventional approach of poles lying near the unit circle in Z-plane but by taking residue to pole ratio (Gupta and Kumar, 2016). The higher the value of residue to pole ratio, the more dominant the pole is (Singh, et al., 2004). The selection of dominant pole is based on the ratio of absolute value of residue to absolute value of real part of the poles (Takahashi, et al., 1987). This method of selecting poles results in an improved approximation. The implementation of pole clustering techniques has made the model conceptually simple and easy to control through pole clustering (Sinha and Pal, 1990). A single sixth order system have been used and reduced to $2^{\text {nd }}$ order approximant and then the response of the original and reduced models have been compared by plotting step response. With the fast advancement and innovations in small digital computers and processors, in the implementation and design of control system promotes the importance of model order reduction methods for discrete time systems. The Desirable reduction methods should have qualities of good approximation along with low cost, minimum computational time and lesser memory requirements.

Kumar and Chandra (2014) have proposed the Padé approximants for reduction of higher order system in continuous-time domain (Hutton and Friedland, 1975) and discrete-time system have been suggested later in this paper in order to achieve stable model. Model order reduction by means of matching Markov parameters, impulse energy approximation and time moments approximation confirms the stability of the reduced order model (Choo, 2006) An easy model order reduction procedure is suggested for z-transfer functions or for discrete time domain that uses estimated frequency response matching among the original system and the reduced-order model. Many literatures have discussed the reduction procedure in this domain but the technique applied in this paper is original.

The investigator proposes to obtain a reduced order model of a discrete time system by incorporating the dominancy criteria to the pole clustering technique. A new approach has been adopted for cluster formation based on the dominancy criteria to determine the denominator polynomial while numerator of reduced order model has been obtained by Padé Approximation. (Singh and Chandra, 2012) The result obtained from the proposed method has been compared with the conventional way of reduction. The result shows the superiority of the proposed approach.

\section{Model Order Reduction Methods}

Many methods have been established for minimising the large scale system analysing the behaviour of the reduced model with the original which can be classified in general in two steps.

\subsection{Classical Reduction Method (CRM)}

This method is based on the conventional concepts of mathematical approximation such as Padéapproximation, continued-fraction method and time-moments matching method (Mukherjee and Mittal, 2005) and all these are equivalent to each other. 
International Journal of Mathematical, Engineering and Management Sciences

Vol. 4, No. 1, 56-65, 2019

https://dx.doi.org/10.33889/IJMEMS.2019.4.1-005

There is some limitation to this method such as

(i) Even though the original system is a stable reduced model may be unstable.

(ii) Low accuracy in frequencies of mid and high ranges.

\subsection{Dominant Pole Based Clustering Reduction (DPBCR)}

Dominant pole based pole clustering methodology for obtaining model order reduction is presented (Kumar and Chandra, 2014). The method uses dominance by determining the value of the ratio of the residue to real parts of poles, taken in descending order. An example is included to show the improvement in system approximation by present approach over conventional approaches.

\section{Problem Statement}

Consider a linear SISO $n^{\text {th }}$ order system transfer function

$\mathrm{G}(\mathrm{z})=\frac{\mathrm{N}(\mathrm{z})}{\mathrm{D}(\mathrm{z})}=\frac{\mathrm{a}_{\mathrm{n}}+\mathrm{a}_{\mathrm{n}-1} \mathrm{z}+\cdots+\mathrm{a}_{1} \mathrm{z}^{\mathrm{n}-1}}{\mathrm{~b}_{\mathrm{n}}+\mathrm{b}_{\mathrm{n}-1} \mathrm{z}+\cdots+\mathrm{b}_{1} \mathrm{z}^{\mathrm{n}-1}+\mathrm{z}^{\mathrm{n}}}$

We prefer to obtain an $r^{\text {th }}$ order reduced model such that $r<n$ given by

$\widehat{G}(p)=\frac{\widehat{N}(p)}{\widehat{D}(p)}=\frac{\hat{a}_{r}+\widehat{a}_{r-1} p+\cdots+\widehat{a}_{1} p^{r-1}}{\widehat{b}_{r}+\widehat{b}_{r-1} p+\cdots+\widehat{b}_{1} p^{r-1}+p^{r}}$

Suppose the specified system be expressed as

$\mathrm{G}(\mathrm{z})=\sum_{\mathrm{i}=1}^{\mathrm{n}} \frac{\mathrm{R}_{\mathrm{i}}}{\mathrm{z}-\lambda_{\mathrm{i}}}$

With the use of Eq. (3) corresponding to each pole $\lambda_{i} a$ fraction of residue to pole given by $\frac{\left|R_{i}\right|}{\left|\operatorname{Re}\left(\lambda_{i}\right)\right|}$ is calculated. Then the pole $\lambda_{i}$ is sorted in their decreasing value of $\frac{\left|R_{i}\right|}{\left|\operatorname{Re}\left(\lambda_{i}\right)\right|}$. After that the $n$-poles $\left(\lambda_{1}, \lambda_{2}, \lambda_{3}, \ldots . \lambda_{n}\right)$ are collected in $r$ clusters such that the number of poles is equal in either all the clusters or in maximum numbers of cluster. The dominant pole is decided by the criteria given in the corollary 1.

\section{Corollary 1}

A pole $\lambda_{i}$ is called dominant pole if $\frac{\left|R_{i}\right|}{\left|\operatorname{Re}\left(\lambda_{i}\right)\right|}>\frac{\left|R_{j}\right|}{\left|\operatorname{Re}\left(\lambda_{j}\right)\right|} \forall i, j=1,2 \ldots \ldots, n, i \neq j$. Thus, the first cluster consists of the most dominant set of poles and the next cluster consists of the next most dominant set of poles and so on. After the formation of the clusters, each cluster is considered for pole clustering to obtain the cluster centre separately.

Let $k$ real poles in one cluster group be $\left(p_{1}, p_{2}, p_{3} \ldots p_{k}\right)$ then cluster centre using IDM (inverse distance measurement) criteria is obtained by the Algorithm as

Step -3.1 arrange the poles as $\left|p_{1}\right|<\left|p_{2}\right|<\ldots<\left|p_{k}\right|$.

Step -3.2 Set $i=1$.

Step - 3.3 Find pole cluster as $C_{i}=\left[\sum_{j=1}^{k}\left(\frac{-1}{p_{j}}\right) \div k\right]^{-1}$.

Step -3.4 Increments $i=i+1$. 
International Journal of Mathematical, Engineering and Management Sciences

Vol. 4, No. 1, 56-65, 2019

https://dx.doi.org/10.33889/IJMEMS.2019.4.1-005

Step -3.5 improved pole cluster centre is calculated as $C_{i}=\left[\left(\frac{-1}{p_{1}}\right)+\left(\frac{-1}{p_{i-1}}\right) \div 2\right]^{-1}$.

Step -3.6 Is $k=i$ ? If No, go to step -3.4 otherwise stop.

Let $k$ pair of complex conjugate poles in one cluster group be $\left(A \pm j B_{1}, A_{2} \pm j B_{2}, \ldots, A_{k} \pm\right.$ $\mathrm{jB}_{\mathrm{k}}$ ), where $\left|A_{1}\right|<\left|A_{2}\right|<\ldots<\left|A_{k}\right|$ then cluster centre is obtained using the same procedure as described above, separately for real and imaginary parts of the complex conjugate poles which is given as $A \pm j B_{i}$.

If the poles occur to lie on the imaginary axis, it should be retained. Since the $r^{\text {th }}$ order model is being obtained, $r$ cluster centres are obtained. Depending on the nature of poles the following three cases may occur.

\section{Case-1}

If cluster centres obtained are all real, the denominator takes the form as

$\widehat{D}(p)=\prod_{i=1}^{r}\left(p-A_{i}\right)$

\section{Case-2}

If cluster centres obtained are all complex conjugate, the denominator takes the form as

$\widehat{D}(p)=\prod_{i=1}^{r / 2}\left[p-\left(A_{i}-j B_{i}\right)\right]\left[p-\left(A_{i}+j B_{i}\right)\right]$

\section{Case-3}

If few cluster centres (say 2) obtained is real and remaining cluster centres are complex conjugate, the denominator takes the form as

$$
\widehat{D}(p)=\left(p-A_{1}\right)\left(p-A_{2}\right) \prod_{i=3}^{(r-2) / 2}\left[p-\left(A_{i}-j B_{i}\right)\right]\left[p-\left(A_{i}+j B_{i}\right)\right]
$$

The denominator so obtained tries to retain the most dominant feature of the high order system leading to a far superior approximation. Here $r$ is the order of reduced model considered even but the same theory can be extended while considering $r$ as odd. After determining the denominator the numerator coefficient can be obtained using Padé approximation technique (Figure 1).

\subsection{Computation of Numerator Polynomial}

The numerator polynomial is obtained by matching the time moments of the Taylor series expansion around $z=0$ of original high order system with those of the reduced order model.

Let (1) and (2) are expanded in Taylor series around as

$G(p)=t_{0}+t_{1} p+t_{2} p^{2}+t_{3} p^{3}+\cdots$

where $t_{i}(i=1,2 \ldots)$ are time moments.

$\hat{G}(z)=\hat{t}_{0}+\hat{t}_{1}(z-1)+\hat{t}_{2}(z-1)^{2}+\hat{t}_{3}(z-1)^{3}+\cdots$

where $\hat{t}_{i}$ are time moments and $(i=0,1,2 \ldots)$.

We pursue a stable model for which $r$ equations given by

$\hat{t}_{i}-t_{i}=0, i=0,1,2, \ldots . r$ 
International Journal of Mathematical, Engineering and Management Sciences

Vol. 4, No. 1, 56-65, 2019

https://dx.doi.org/10.33889/IJMEMS.2019.4.1-005

are satisfied which implies that

$\hat{a}_{r+1-i}=\sum_{j=1}^{i} \hat{t}_{j} \hat{b}_{r-i+j}, \quad i=0,1,2,3 \ldots r$

Thus, coefficients of numerator polynomial of (2) can be obtained using (10). The above mentioned procedure is illustrated in the next section through an example taken from the literature.

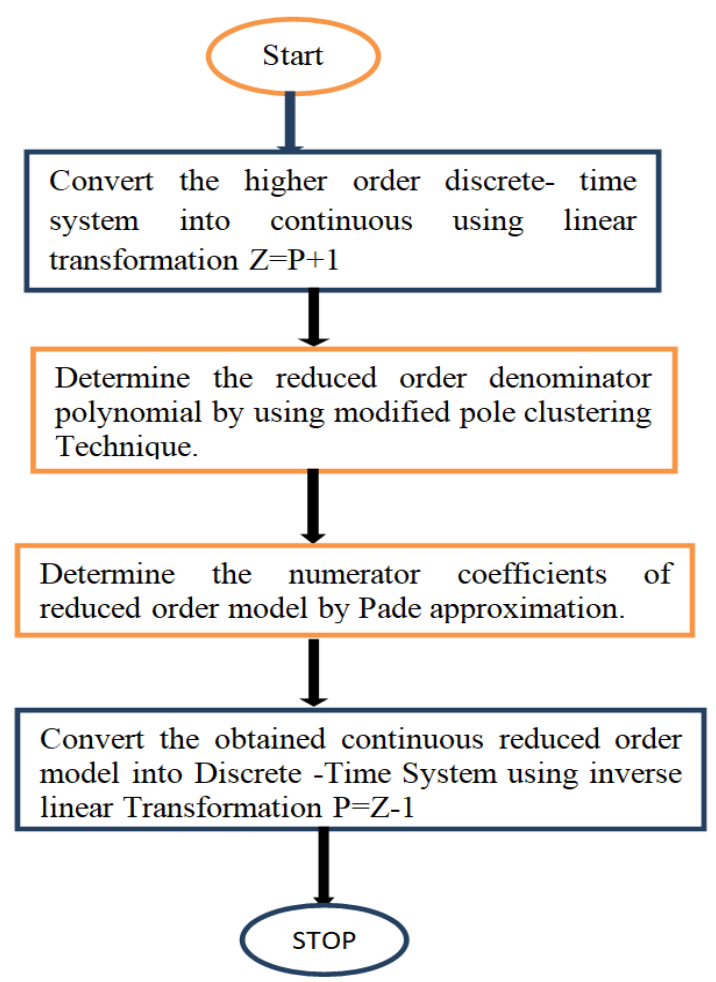

Figure 1. Flow chart of step wise procedure for computation

\section{Model Order Reduction Procedure Numerical Example}

Firstly calculating the time moments of the system and then Padé approximation problem is formulated. Consider the transfer function of the higher order discrete time system is expressed as $\mathrm{G}(\mathrm{z})=\frac{\mathrm{a}_{1} \mathrm{z}^{\mathrm{n}-1}+\mathrm{a}_{2} \mathrm{z}^{\mathrm{n}-2}+\cdots \ldots \ldots \ldots+\mathrm{a}_{\mathrm{n}}}{\mathrm{z}^{\mathrm{n}}+\mathrm{b}_{1} \mathrm{z}^{\mathrm{n}-1}+\cdots \ldots \ldots \ldots \ldots+\mathrm{b}_{\mathrm{n}}}$

Assume that a reduced order model of the form

$\mathrm{G}_{\mathrm{r}}(\mathrm{z})=\frac{\mathrm{a}_{1} \mathrm{z}^{\mathrm{r}-1}+\mathrm{a}_{2} \mathrm{z}^{\mathrm{r}-2}+\cdots \ldots \cdots \cdots \cdots \cdots+\mathrm{a}_{\mathrm{r}}}{\mathrm{z}^{\mathrm{r}}+\mathrm{b}_{1} \mathrm{z}^{\mathrm{r}-1}+\cdots \ldots \cdots \cdots \cdots \cdots \cdots+\mathrm{b}_{\mathrm{r}}}$

Eq.(12) is to be constructed, where $r<n$. the calculations of time moments for the discrete time system are as follows, when equation(12) is converted into continuous domain it will become 
International Journal of Mathematical, Engineering and Management Sciences

Vol. 4, No. 1, 56-65, 2019

https://dx.doi.org/10.33889/IJMEMS.2019.4.1-005

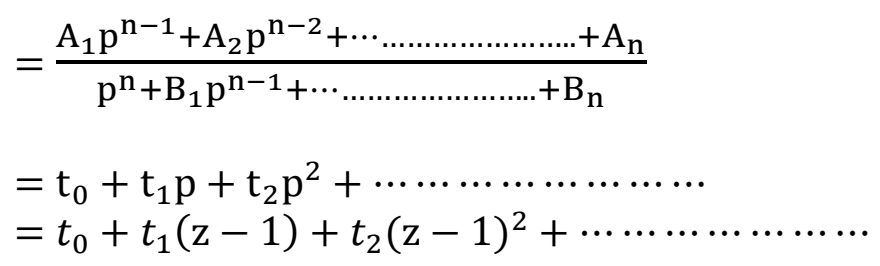

The parameter $t_{i}$ 's are given by

$\mathrm{t}_{0}=\frac{\mathrm{A}_{\mathrm{n}}}{\mathrm{B}_{\mathrm{n}}}$

$t_{i}=\left(A_{n-i}-\sum_{j=0}^{i=1} B_{n+j-i} t_{j}\right) / B_{n}, \mathrm{i}=1,2, \ldots \ldots$

Consider a sixth order system

$$
G(z)=\frac{2.04(z-0.75)(z-0.9423592)(z-0.717847)(z-0.5195656)(z-0.5)}{(z-0.3)(z-0.5)(z-0.75)(z-0.85)(z-0.9)(z-0.95)}
$$

and suppose a second order approximant of the form $G_{r}(z)=\frac{a_{1} z+a_{2}}{z^{2}+b_{1} z+b_{2}}$ is required.

Substituting $z=p+1$ in sixth order system yields $G(p)=\frac{n u m}{d e n}$

$n u m=2.04 p^{5}+3.203998 p^{4}+1.877097 p^{3}+5001582 p^{2}+0.0577944 p+$ 0.0019949

den $=p^{6}+1.75 p^{5}+1.1125 p^{4}+0.323125 p^{3}+0.45212 p^{2}+0.002893 p+$ 0.0000656

The residues $(r)$, poles $(p)$ and gain $(k)$ of the equation (15) and (16) is

$r=0.5605,0.0000,-0.0000,-2.9919,3.9921,0.4793$

$p=-0.7000,-0.5000,-0.2500,-0.1500,-0.1000,-0.0500$

$k=[]$.

The poles are arranged according to the classical approach so as to compare the result with the original model and to compute the denominator of the reduced order model. The $2^{\text {nd }}$ order reduced model is required to be realized. Based on the classical dominance criteria the clusters are formed as

Cluster-1: poles $(-0.05,-0.1,-0.15,-0.25)$.

Cluster-2: poles $(-0.5,-0.7)$.

Cluster centres obtained using the same algorithm as given in step 3.3 and step 3.5 is $(-0.06623,-0.54)$. 
International Journal of Mathematical, Engineering and Management Sciences

Vol. 4, No. 1, 56-65, 2019

https://dx.doi.org/10.33889/IJMEMS.2019.4.1-005

Hence, the denominator polynomial is given as

$(p+0.54)(p+0.06623)=p^{2}+0.61 p+0.036$.

The denominator polynomial is calculated and for numerator polynomial, we have to calculate time moments from the original system obtained after substitution

$t_{0}=30.4101$

$t_{1}=-460.0917576$

$t_{2}=-181673.5809$.

Now substituting $\mathrm{z}=p+1$ in reduced order model gives

$G_{r}(p)=\frac{a_{1} p+\left(a_{1}+a_{2}\right)}{p^{2}+\left(2+b_{1}\right) p+\left(1+b_{1}+b_{2}\right)}$

Where $t_{0}=\frac{a_{1}+a_{2}}{1+b_{1}+b_{2}}$ and $t_{1}=\frac{a_{1}-t_{0}\left(2+b_{1}\right)}{1+b_{1}+b_{2}}$

Using the equation (17), (18) and denominator polynomial i.e., $p^{2}+0.61 p+0.036$ and time moments we get the numerator coefficient as $1.986561 p+1.0947636$ and the transfer

function hence obtained is $G_{p 1}=\frac{1.986561 \mathrm{p}+1.0947636}{\mathrm{p}^{2}+0.61 \mathrm{p}+0.036}$

And after substituting $p=\mathrm{z}-1$ we get

$G_{r 1}=\frac{1.986561 \mathrm{z}-0.8917974}{\mathrm{z}^{2}-1.39 \mathrm{z}+0.436}$

Eq. (20) is the reduced order model obtained by the classical approach.

Now we are going to calculate the reduced order transfer function based on the dominancy criteria of ratio of residue to poles using (DPBCA) approach.

For sorting the poles based on their dominance, the ratio of absolute value of residue $\left(R_{i}\right)$ to the corresponding absolute value of the real part of the poles $\left(\operatorname{Re}\left(\lambda_{i}\right)\right)$ is computed.

The Table 1 shows the poles with the corresponding ratio $\frac{\left|R_{i}\right|}{\left|\operatorname{Re}\left(\lambda_{i}\right)\right|}$. 
International Journal of Mathematical, Engineering and Management Sciences

Vol. 4, No. 1, 56-65, 2019

https://dx.doi.org/10.33889/IJMEMS.2019.4.1-005

Table 1. Ratio of residue to poles

\begin{tabular}{|c|c|c|}
\hline S. No. & Poles & $\frac{\left|\boldsymbol{R}_{\boldsymbol{i}}\right|}{\left|\boldsymbol{R e}\left(\boldsymbol{\lambda}_{\boldsymbol{i}}\right)\right|}$ \\
\hline 1 & -0.7 & $\frac{0.5605}{0.7}=0.8$ \\
\hline 2 & -0.5 & $\frac{0.0000}{0.5}=0$ \\
\hline 3 & -0.25 & $\frac{0.000}{0.25}=0$ \\
\hline 4 & -0.15 & $\frac{2.9919}{0.1500}=19.946$ \\
\hline 5 & -0.1 & $\frac{3.9921}{0.1000}=39.921$ \\
\hline 6 & -0.05 & $\frac{0.4793}{0.0500}=9.586$ \\
\hline
\end{tabular}

Based on the dominance criteria as mentioned in corollary- 1 the sorted poles are $-0.05,-0.15,-0.1,-0.7,-0.5,-0.25$.

Let the second order reduced model is required to be realized. For this purpose two clusters are required. Hence by DPPCA the clusters are formed as

Cluster-1: poles $(-0.05,-0.15,-0.1,-0.7)$.

Cluster-2: poles $(-0.5,-0.25)$.

Cluster centre as obtained by the algorithm in the section are $(-0.102435,-0.29)$.

Hence the denominator polynomial is

$(p+0.102435)(p+0.29)=p^{2}+0.392435 p+0.02970615$

Now the numerator polynomial is calculated by using the transfer function $G_{r}(p)$, denominator polynomial obtained above, equation (18) and time moments same as done in classical approach $t_{0}=30.4101, t_{1}=-460.0917576, t_{2}=-181673.5809$

the transfer function comes out to be

$G_{p 2}=\frac{-1.733812027 p+0.903366992}{p^{2}+0.392435 p+0.02970615}$

and after substituting $p=\mathrm{z}-1$ we get $G_{r 2}=\frac{-1.733812027 \mathrm{z}+2.637179019}{\mathrm{z}^{2}-1.607565 \mathrm{z}+0.63727115}$ 
International Journal of Mathematical, Engineering and Management Sciences

Vol. 4, No. 1, 56-65, 2019

https://dx.doi.org/10.33889/IJMEMS.2019.4.1-005

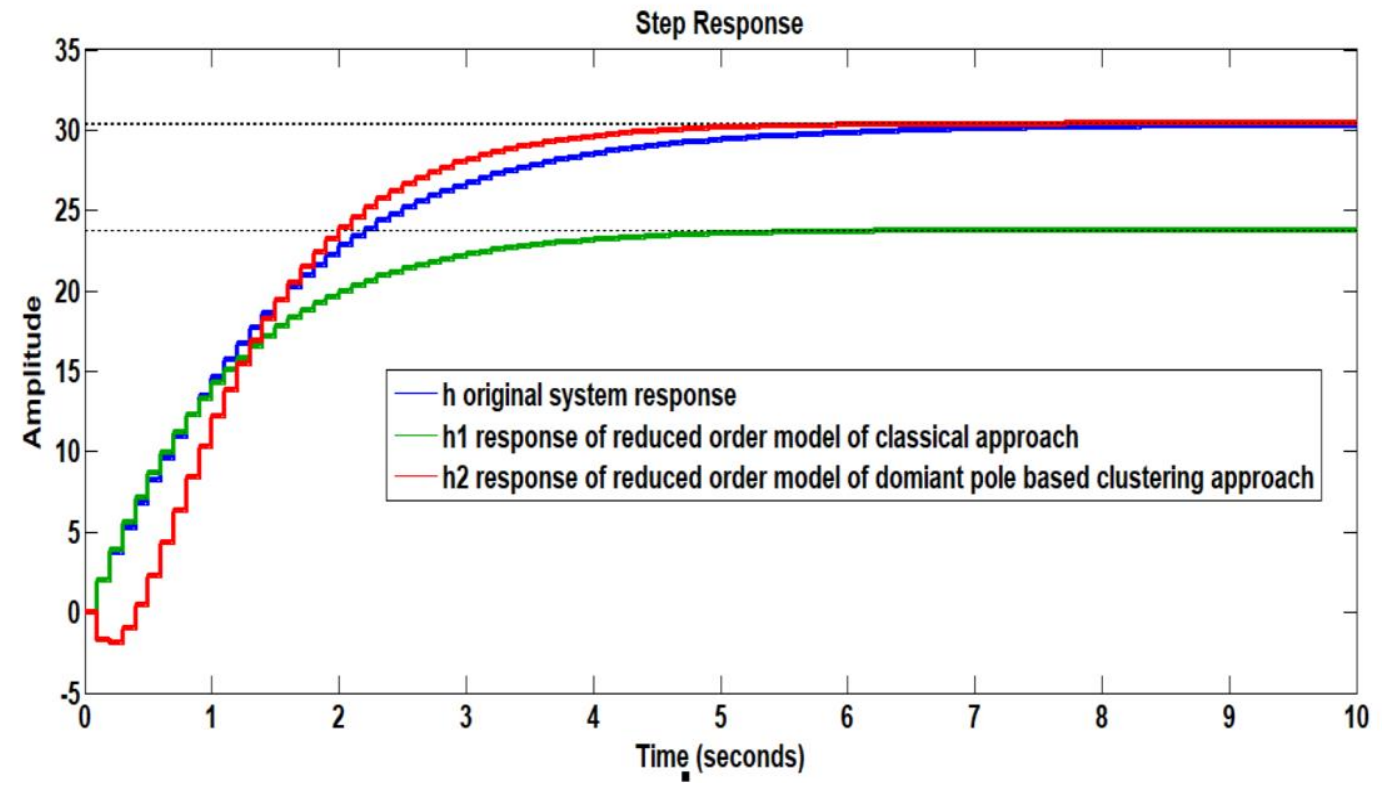

Figure 2. Step response of the original higher order system and reduced order system

Eq. (22) is the reduced order model obtained using Dominant Pole Based Clustering (DPBCA) approach.

In Figure 2, step response of the original higher order system and the two reduced models are shown which reveals that DPBCA is a good approximant.

\section{Conclusion}

A method based on Dominant Pole Based Clustering Approach (DPBCA) using Padé Approximation Technique and Time Moment Matching of parameters along with the dominance of $\mathrm{z}$ plane has been used to reduce the model of higher order discrete time system. In this paper, a novel approach for deriving a reduced order model using DPBCA is presented. In this work, modified pole clustering is used for determination of denominator polynomial and Padé approximation is used for obtaining numerator coefficients of the reduced order model. The cluster formation has been taken based on the Dominant Pole Based Clustering Approach (DPBCA). The method assures the stability of the system with a reduced order model. With the help of a selected example from literature, the efficacy of proposed method is clarified and response of the original and reduced system has been graphically compared which shows the superiority of the approach.

\section{Conflict of Interest}

The authors confirm that there is no conflict of interest to declare for this publication.

\section{Acknowledgement}

The authors would like to thank reviewers for their constructive comments and for their valuable suggestions towards the improvement of the paper. 
International Journal of Mathematical, Engineering and Management Sciences

Vol. 4, No. 1, 56-65, 2019

https://dx.doi.org/10.33889/IJMEMS.2019.4.1-005

\section{References}

Bistritz, Y. U. V. A. L., \& Shaked, U. (1984). Discrete multivariable system approximations by minimal Padé-type stable models. IEEE Transactions on Circuits and Systems, 31(4), 382-390.

Choo, Y. (2006). Suboptimal bilinear Routh approximant for discrete systems. Journal of Dynamic Systems, Measurement, and Control, 128(3), 742-745.

Gupta, M. K., \& Kumar, A. (2016). Model reduction of continuous and discrete time systems using differentiation method with many clustering techniques. i-Manager's Journal on Instrumentation \& Control Engineering, 4(3), 27.

Hutton, M., \& Friedland, B. (1975). Routh approximations for reducing order of linear, time-invariant systems. IEEE Transactions on Automatic Control, 20(3), 329-337.

Kumar, A., \& Chandra, D. (2014). Improved Padé-Pole clustering approximant. In International Conference on Computer Science and Electronics Engineering, pp. 28-32.

Mukherjee, S., \& Mittal, R. C. (2005). Model order reduction using response-matching technique. Journal of the Franklin Institute, 342(5), 503-519.

Ramesh, K., Ganesan, R. G., \& Mahalakshmi, K. (2017). Approximation and optimization of discrete systems using order reduction technique. Energy Procedia, 117, 761-768.

Sinha, A. K., \& Pal, J. (1990). Simulation based reduced order modelling using a clustering technique. Computers \& Electrical Engineering, 16(3), 159-169.

Singh, V., Chandra, D., \& Kar, H. (2004). Improved Routh-Pade/spl acute/approximants: a computer-aided approach. IEEE Transactions on Automatic Control, 49(2), 292-296.

Shamash, Y. (1974). Stable reduced-order models using Padé-type approximations. IEEE Transactions on Automatic Control, 19(5), 615-616.

Singh, V. P., \& Chandra, D. (2012). Model reduction of discrete interval system using clustering of poles. International Journal of Modelling, Identification and Control, 17(2), 116-123.

Takahashi, S., Yamanaka, K., \& Yamada, M. (1987). Detection of dominant poles of systems with time delay by using Padé approximation. International Journal of Control, 45(1), 251-254.

Vishwakarma, C. B., \& Prasad, R. (2008). System reduction using modified pole clustering and Pade approximation. XXXII National Systems Conference, NSC 2008, December 17-19, 2008. 